\title{
Representations of quivers over finite fields
}

\section{JIUZHAO HUA}

Let $\mathbb{F}_{q}$ be the finite field of $q$ elements. Let $\Gamma$ be a connected graph with vertices $\{1,2, \cdots, n\}$, and let $a_{i j}$ be the number of edges connecting $i$ and $j$. Let $\Delta \subset \mathbb{Z}^{n}$ be the root system associated with $\Gamma$. For any $\alpha \in \mathbb{N}^{n}$, let $A_{\Gamma}(\alpha, q)$ be the number of classes of absolutely indecomposable representations of $\Gamma$ (with respect to a fixed orientation) over $\mathbb{F}_{q}$ with dimension $\alpha$. A theorem of Kac asserts that $A_{\Gamma}(\alpha, q) \neq 0$ if and only if $\alpha \in \Delta^{+}$. It is known that $A_{\Gamma}(\alpha, q)$ is a polynomial in $q$ with integer coefficients; these have been conjectured to be non-negative by Kac. Thus, we may assume that $A_{\Gamma}(\alpha, q)=\sum_{i=0}^{u_{\alpha}} t_{i}^{\alpha} q^{i}$ with $t_{i}^{\alpha} \in \mathbb{Z}$.

Let $\mathcal{P}$ denote the set of all partitions. For $\lambda \in \mathcal{P}$, we let $\lambda^{\prime}=\left(\lambda_{1}^{\prime}, \lambda_{2}^{\prime}, \cdots\right)$ denote the partition conjugate to $\lambda$. For any $\lambda, \mu \in \mathcal{P}$, we define $\langle\lambda, \mu\rangle=\sum_{i \geqslant 1} \lambda_{i}^{\prime} \mu_{i}^{\prime}$. For any $\lambda=\left(1^{n_{1}} 2^{n_{2}} \cdots\right) \in \mathcal{P}$, we define $b_{\lambda}(q)=\prod_{i \geqslant 1}(1-q)\left(1-q^{2}\right) \cdots\left(1-q^{n_{i}}\right)$. Let $X_{1}, \cdots, X_{n}$ be $n$ independent commuting variables, and for $\alpha=\left(\alpha_{1}, \cdots, \alpha_{n}\right) \in \mathbb{Z}^{n}$, we set $X^{\alpha}=X_{1}^{\alpha_{1}} \cdots X_{n}^{\alpha_{n}}$.

The main result in this thesis can be stated as the following formal identity:

$$
\sum_{\lambda_{1}, \cdots, \lambda_{n} \in \mathcal{P}} \frac{\prod_{1 \leqslant i \leqslant j \leqslant n} q^{a_{i j}\left\langle\lambda_{i}, \lambda_{j}\right\rangle}}{\prod_{1 \leqslant n} q^{\left(\lambda_{i}, \lambda_{i}\right\rangle} b_{\lambda_{i}}\left(q^{-1}\right)} X_{1}^{\left|\lambda_{1}\right|} \cdots X_{n}^{\left|\lambda_{n}\right|}=\prod_{\alpha \in \Delta+} \prod_{i=0}^{\infty} \prod_{j=0}^{u_{\alpha}}\left(1-q^{i+j} X^{\alpha}\right)^{t_{j}^{\alpha}}
$$

Kac has conjectured that if $\Gamma$ does not contain edge-loops then the constant term of $A_{\Gamma}(\alpha, q)$ equals the multiplicity of $\alpha$, which is defined to be the dimension of the root space corresponding to $\alpha$ of the Kac-Moody algebra determined by $\Gamma$. By assuming this conjecture, the above identity turns out to be a $q$-analogue of the Weyl-Macdonald-Kac denominator identity.

Another result in this thesis is as follows. Let $A$ be a finite dimensional algebra over a perfect field $\mathbb{K}$, and let $M$ be a finitely generated indecomposable module over

Received 24th September, 1998

Thesis submitted to the University of New South Wales, February 1998. Degree approved, July 1998. Supervisor: Peter Donovan.

Copyright Clearance Centre, Inc. Serial-fee code: 0004-9729/99 \$A2.00+0.00. 
$A \otimes_{\mathbb{K}} \overline{\mathbb{K}}$, where $\overline{\mathbb{K}}$ denotes the algebraic closure of $\mathbb{K}$. If there exists a module $N$ over $A \otimes_{\mathbb{K}} \mathbb{E}$, where $\mathbb{E}$ is a finite extension of $\mathbb{K}$, such that $M \cong N \otimes_{\mathbb{E}} \overline{\mathbb{K}}$, then $\mathbb{E}$ is called a field of definition of $M$. It is proved that for each $M$ there exists a unique indecomposable module $M^{\dagger}$ over $A$ such that $M$ is a direct summand of $M^{\dagger} \otimes_{\mathbf{K}} \overline{\mathbb{K}}$, and there exists a positive integer $s$ such that $M^{s}=M \oplus \cdots \oplus M$ (s copies) has a unique minimal field of definition which is isomorphic to the centre of the division algebra $\left(\operatorname{End}_{\Gamma}\left(M^{\dagger}\right)\right) /\left(\operatorname{rad}\left(\operatorname{End}_{\Gamma}\left(M^{\dagger}\right)\right)\right)$. If $\mathbb{K}$ is a finite field, then $s$ can be taken to be 1 .

Part of this thesis has already been published in [1].

\section{REFERENCES}

[1] J. Hua, 'Generalizing the recursion relationship for the partition function', $J$. Combin. Theory Ser. A 79 (1997), 105-117.

School of Mathematics

University of NSW

Sydney NSW 2052

Australia

e-mail: hua@maths.unsw.edu.au 